\title{
Analysis of Gender Difference of Cardiac Risk Biomarkers Using hGH-Transgenic Mice
}

\author{
Hitoshi NARAOKA ${ }^{1)}$, Kyoko ITO'), Michie SUZUKI'), \\ Kunihiko NAITO ${ }^{1}$, and Hideaki TOJO ${ }^{1)}$ \\ 1)Laboratory of Applied Genetics, Graduate School of Agricultural and Life Sciences, \\ The University of Tokyo, 1-1-1 Yayoi, Bunkyo-ku, Tokyo 113-8657, and \\ ${ }^{2}$ Safety Research Laboratories, Yamanouchi Pharmaceutical Co Ltd. \\ 1-1-8 Azusawa, Itabashi-ku, Tokyo 174-8511, Japan
}

\begin{abstract}
We investigated gender difference in the effects of chronic exposure to human growth hormone $(\mathrm{hGH})$ on cardiac risk biomarkers using transgenic mice with non-pulsatile circulating $h \mathrm{GH}$. Blood plasma was obtained from transgenic and control mice at 8,12 , and 16 weeks of age, and was used for the measurement of $h G H$ and the following cardiac risk biomarkers: total cholesterol (CHO), triglyceride (TG), HDL cholesterol (HDL), LDL cholesterol (LDL), non esterified free fatty acids (NEFA), and lipid peroxides (LPO). The hearts and the livers of transgenic mice were weighed and histopathologicaly examined, and the results were compared with those of control mice. Transgenic males exhibited higher levels of LDL at 8 and 12 weeks of age and higher levels of LPO at every week of age examined, as compared to those of the control males, while transgenic females exhibited somewhat lower levels of LDL and LPO from 8 to 16 weeks of age, as compared to the control females. The relative heart weight in males increased with aging and was significantly higher in the 16-week-old transgenic males compared to those of the control mice. The present results demonstrate that transgenic males had cardiac risk potential caused by chronic-exposure to hGH as compared to females. The results also show that the present transgenic mouse line is a useful model for the study of gender difference in cardiac disorders caused by $h \mathrm{GH}$.
\end{abstract}

Key words: cardiac risk, gender difference, human growth hormone, transgenic mice

\section{Introduction}

Human growth hormone (hGH) is the most abundant hormone secreted from the pituitary gland. GH is known to regulate protein, glucose, and lipid metabolism, in addition to promoting growth. Therefore, hGH has been used as a therapeutic medicine, not only among patients with GH deficiency (GHD), but also among those with heart disease [7]. It has been reported that patients with acromegaly or GHD frequently have concomitant cardiac disorders $[3,26]$. Therefore, it is important to carefully consider the cardiac risk caused

(Received 14 July 2005 / Accepted 6 October 2005)

Address corresponding: H. Tojo, Laboratory of Applied Genetics, Graduate School of Agricultural and Life sciences, The University of Tokyo, 1-1-1 Yayoi, Bunkyo-ku, Tokyo 113-8657, Japan 
by the chronic use of hGH, when hGH is exogenously administered. Furthermore, it is generally known that coronary heart disease develops earlier in males than in females, and its incidence is higher in males [2, 5]. Recently, reports have been published about the importance of therapy accommodating gender difference [16, 25]. However, the gender differences in cardiac disorders caused by GH is not well clarified because there have been few reports regarding these diseases. In addition, the chronic use of the administration of biotechnology-derived pharmaceuticals to laboratory animals is associated with numerous problems, including the production of neutralizing antibody in animals. Therefore, an animal model is necessary for resolving these problems.

To circumvent the neutralization of exogenous hGH by host antibody, transgenic mice that overexpress hGH have been widely used to study the effects of excessive levels of peripheral GH. We previously generated transgenic mice bearing a CAG/EGFP-WAP/hGH cassette gene to investigate the in vitro selection of transgenic embryos prior to embryo transfer [17]. This transgenic mouse line ubiquitously expresses EGFP under the control of the CAG promoter [22], and expresses hGH under the control of the WAP promoter in a tissue-specific manner. This strain of mouse can also be utilized to examine circulating hGH [17], therefore, we used this transgenic mouse line to investigate gender difference in biomarkers for cardiac risk caused by abnormal levels of hGH.

\section{Materials and Methods}

\section{Mice}

The transgenic mouse line used in the present study carries a cassette gene of CAG/EGFP and $\mathrm{WAP} / \mathrm{hGH}$, in which EGFP is ubiquitously expressed under the control of the CAG promoter, and hGH is expressed under the control of the WAP promoter (Fig. 1-A): Circulating $\mathrm{hGH}$ is observed in this transgenic strain [17]. These transgenic mice have been backcrossed at Yamanouchi Pharmaceutical's Safety Research Laboratories for more than five generations into a $\mathrm{C} 57 \mathrm{BL} / 6 \mathrm{~J}$ background. C57BL/6J mice were purchased from CLEA Japan, Inc. (Tokyo, Japan) and were used as controls. The room in which the animals were housed (conventional grade) was air-conditioned at $23 \pm 3^{\circ} \mathrm{C}$, and the animals were exposed to a 13-hr/day light cycle from 8:00 to 21:00. The purchased mice were acclimatized for at least 1 week prior to use. Mice were fed a commercial pellet diet (CRF-1, Oriental Yeast Industries, Tokyo, Japan) and were provided with tap water ad libitum. Transgenic mice were distinguishable from the controls by fluorescence of the body when they were exposed to a hand-held illuminator equipped with an ultraviolet filter (Fig. 1-B). The expression of the hGH transgene was confirmed by the measurement of plasma hGH by radioimmunoassay. The body weights of the transgenic and control mice were measured at 8 , 12, and 16 weeks of age. The animal experiments were conducted according to the Guidelines for the Care and Use of Laboratory Animals, Yamanouchi Pharmaceutical, Co., Ltd.

\section{Measurements of clinical chemistry}

At each week of age, animals were anesthetized with ether, and whole blood anticoagulated with heparin lithium was taken from the inferior vena cava with a sterile syringe (Terumo Co. Ltd, Tokyo, Japan). The plasma was obtained by centrifugation $(1870 \times \mathrm{g}$ for 10 min) and was stored at $-80^{\circ} \mathrm{C}$ until use. The concentration of hGH in the plasma was measured by radioimmunoassay (RIA) using the HGH RIAKIT II (Dinabot Co., Ltd., Tokyo, Japan). The plasma clinical chemistry was analyzed using a 7170 type autoanalyzer (Hitachi Ltd., Tokyo, Japan), the total cholesterol (CHO) by cholesterol oxidase HDAOS assay, triglyceride (TG) by GPO DAOS assay, and non-esterified fatty acid (NEFA) by ACS ACOD assay. HDL cholesterol (HDL) and LDL cholesterol (LDL) were measured by direct assays, and lipid peroxides (LPO) were measured by hemoglobin methylene blue assay. The concentrations of CHO, TG, HDL, and LDL were measured in $\mathrm{mg} / \mathrm{dl}$, the NEFA concentration was measured in $\mathrm{mEq} / \mathrm{l}$, and the LPO concentration was measured in $\mathrm{nmol} / \mathrm{ml}$. The reagents used for the assays of $\mathrm{CHO}, \mathrm{TG}, \mathrm{HDL}, \mathrm{LDL}$, and NEFA were obtained from Wako Pure Chemical Industries, Ltd. (Osaka, Japan), and those used for the LPO assay were obtained from Kyowa Medex Co., Ltd. (Tokyo, Japan).

\section{Histopathology}

Immediately after the collection of blood samples, the animals were exsanguinated and necropsied, and 
A
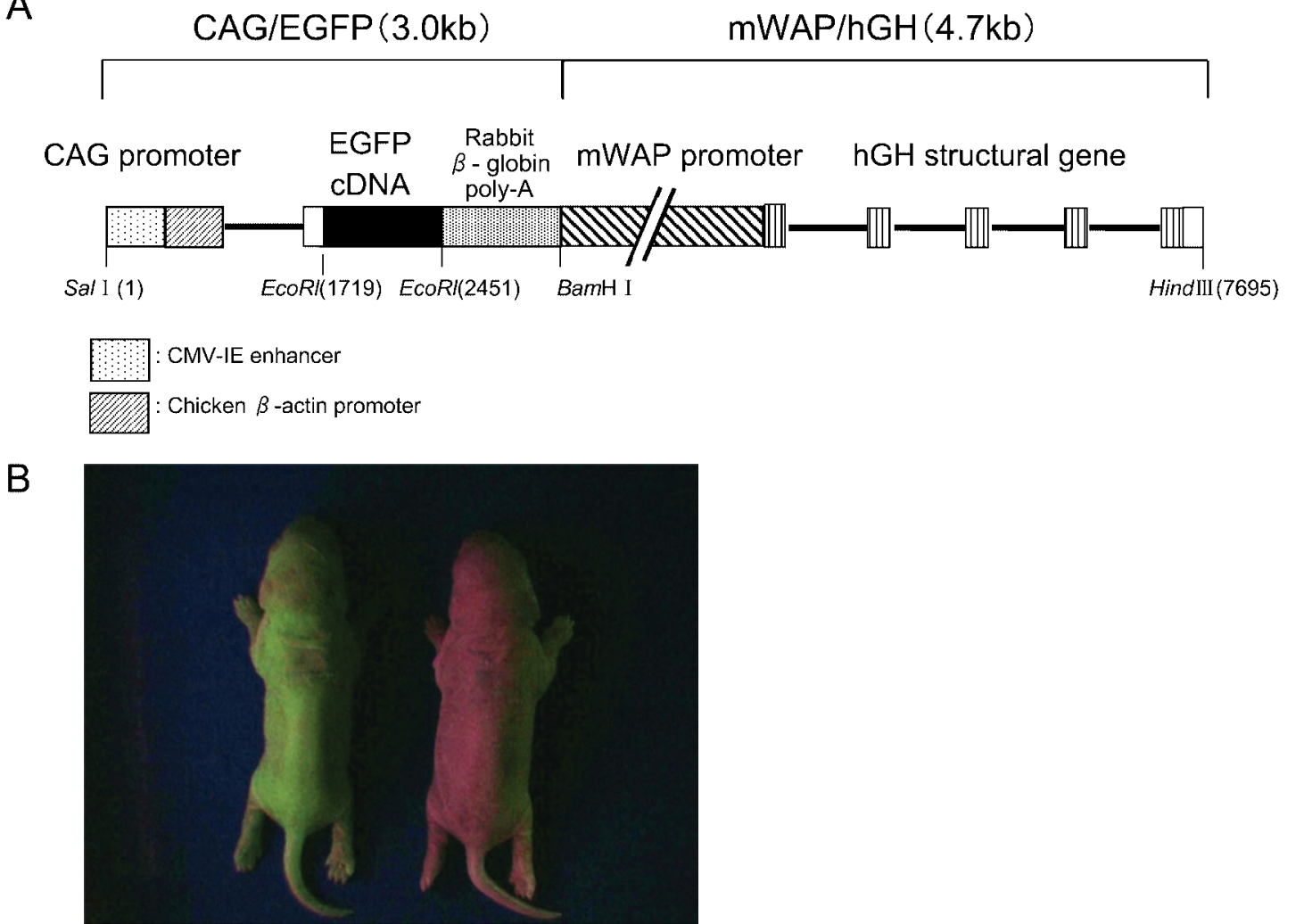

Fig. 1. Schematic structure of the CAG/EGFP-mWAP/hGH cassette gene (A) and photograph of a transgenic pup (B). EGFP, enhanced green fluorescent protein; mWAP, mouse whey acidic protein; hGH, human growth hormone. The transgenic pup (left) expresses EGFP, which can be observed across the entire surface of the body when the pup is placed under UV light; control pup (right).

the tissues were weighed and used for the histological examinations. The hearts and the livers were fixed in phosphate-buffered $10 \%$ formalin and were embedded in paraffin, sectioned at $5 \mu \mathrm{m}$, and stained with hematoxylin and eosin.

\section{Statistical analysis}

The data were analyzed by Student's $t$-test. Differences between the mean values were considered statistically significant at $P<0.05$.

\section{Results}

\section{Body and tissue weights}

No difference in the mean body weight of transgenic males and control males was observed during the period of the experiment when the animals were 8 to 16 weeks of age. The transgenic females showed signifi- cant increases in body weight at every week of age examined, as compared to the controls. The mean weight of the hearts and the livers was significantly greater in the transgenic females than in the controls at every week of age examined, whereas the mean weight was significantly greater in the transgenic males only at 16 weeks of age. The mean weights of the heart in relation to body weight were significantly greater than those of the respective control mice in the transgenic females at 8 weeks of age, and in the transgenic males at 16 weeks of age (Table 1).

\section{Plasma levels of clinical chemistries}

Plasma levels of hGH in the transgenic mice are shown in Table 2, and the data regarding other plasma chemistry results in relation to the values of control mice are shown in Fig. 2. The mean concentration of hGH was higher in the transgenic males than in the 
Table 1. Body and organ weights in the transgenic mice and control mice (C57BL/6J)

\begin{tabular}{|c|c|c|c|c|c|c|c|}
\hline & \multirow{2}{*}{ Sex } & \multirow{2}{*}{$\begin{array}{c}\text { Weeks of } \\
\text { Age }\end{array}$} & \multirow{2}{*}{$\begin{array}{c}\text { Body } \\
\text { Weight }(\mathrm{g})\end{array}$} & \multicolumn{2}{|c|}{ Organ Weight (mg) } & \multicolumn{2}{|c|}{ Relative Organ Weight $(\mathrm{mg} / 100 \mathrm{~g})$} \\
\hline & & & & Liver & Heart & Liver & Heart \\
\hline \multirow[t]{6}{*}{ Control } & M & 8 & $26.1 \pm 0.4$ & $1402 \pm 80$ & $133 \pm 13$ & $5370 \pm 287$ & $510 \pm 44$ \\
\hline & & 12 & $30.2 \pm 1.1$ & $1560 \pm 47$ & $143 \pm 9$ & $5161 \pm 153$ & $474 \pm 13$ \\
\hline & & 16 & $32.0 \pm 1.2$ & $1704 \pm 154$ & $142 \pm 7$ & $5320 \pm 273$ & $445 \pm 11$ \\
\hline & $\mathrm{F}$ & 8 & $21.2 \pm 0.2$ & $1184 \pm 19$ & $106 \pm 5$ & $5574 \pm 51$ & $501 \pm 21$ \\
\hline & & 12 & $22.7 \pm 0.8$ & $1212 \pm 54$ & $120 \pm 6$ & $5339 \pm 50$ & $530 \pm 45$ \\
\hline & & 16 & $23.2 \pm 0.4$ & $1236 \pm 89$ & $125 \pm 11$ & $5324 \pm 290$ & $537 \pm 42$ \\
\hline \multirow[t]{6}{*}{ TG } & M & 8 & $25.1 \pm 1.1$ & $1399 \pm 62$ & $127 \pm 4$ & $5595 \pm 11$ & $504 \pm 9$ \\
\hline & & 12 & $29.5 \pm 1.1$ & $1494 \pm 94$ & $149 \pm 11$ & $5064 \pm 306$ & $505 \pm 34$ \\
\hline & & 16 & $31.4 \pm 3.4$ & $1641 \pm 134$ & $169 \pm 21 *$ & $5237 \pm 190$ & $539 \pm 39 * *$ \\
\hline & $\mathrm{F}$ & 8 & $24.2 \pm 0.9 * *$ & $1362 \pm 112 * *$ & $141 \pm 5^{* *}$ & $5621 \pm 254$ & $584 \pm 22 * *$ \\
\hline & & 12 & $28.4 \pm 1.2^{* * *}$ & $1510 \pm 96^{* *}$ & $149 \pm 11^{* *}$ & $5312 \pm 297$ & $523 \pm 28$ \\
\hline & & 16 & $28.7 \pm 2.5^{* *}$ & $1418 \pm 105^{*}$ & $156 \pm 18^{*}$ & $4970 \pm 510$ & $547 \pm 67$ \\
\hline
\end{tabular}

Values (organ weight $\mathrm{mg}$, relative organ weight $\mathrm{mg} / 100 \mathrm{~g}$ ) indicate the mean \pm S.D. $(\mathrm{n}=3)$. M, males; F, females; **: $P<0.01, *: P<0.05$ between control and transgenic mice.

Table 2. Plasma concentrations of human growth hormone $(\mathrm{hGH})$ in the transgenic mice

\begin{tabular}{lccc}
\hline Sex & \multicolumn{3}{c}{ Weeks of Age } \\
& 8 & 12 & 16 \\
\hline Male & $21.0 \pm 4.4$ & $11.6 \pm 2.6$ & $10.0 \pm 2.0$ \\
Female & $12.7 \pm 4.3$ & $12.3 \pm 3.5$ & $10.5 \pm 4.8$ \\
\hline
\end{tabular}

Values $(\mathrm{ng} / \mathrm{ml})$ indicate the mean \pm S.D. $(\mathrm{n}=3)$.

transgenic females at 8 weeks of age, but these groups did not differ in this respect after they reached 12 weeks of age (Table 2). There was no significant gender difference in the $\mathrm{CHO}$ levels between the transgenic males (110-120\%) of control animals and the transgenic females $(98-115 \%)$, when the animals were 8 to 16 weeks of age. The TG concentrations were lower in the transgenic males (65-100\%) and in the transgenic females (60-78\%), compared to those of the controls when the animals were 8 and 16 weeks of age; a significant gender difference was observed at 12 weeks of age. Higher levels of HDL were observed in both the transgenic males (110-127\%) and females (103-132\%) during the course of the entire experiment, however, there was no gender difference in HDL levels. The LDL levels were higher in the transgenic males (114$128 \%$ ), than in the control males, whereas the LDL levels were somewhat lower in the transgenic females
(75-88\%) than in the control females, and a significant gender difference was observed at 8 and 12 weeks of age. NEFA levels were lower in the transgenic males at 8 weeks of age, but higher NEFA levels were observed in the transgenic males at $12(133 \%)$ and 16 $(115 \%)$ weeks of age. On the other hand, among the transgenic females, the NEFA values were significantly high $(152 \%)$ compared to those of the controls at 8 weeks of age, and then decreased to the values of the controls with increasing age. Therefore, a significant gender difference was observed only at 8 weeks of age. In contrast, very unique changes in LPO levels were observed in the transgenic mice, namely, the transgenic males exhibited normal LPO levels at 8 weeks of age (73\%), but extremely high levels were observed in these mice at 12 and 16 weeks of age (400-500\%). However, the LPO levels of the transgenic females were far lower (14-30\%) than those of the female controls during the entire experiment (Fig. 2). Consequently, a significant gender difference was observed during the entire experiment.

\section{Histopathology}

In contrast to the values of the controls, significant increases in the mean weight of the liver and the heart of transgenic mice was observed. We thus examined these tissues histologically. The histopathological data regarding these tissues are shown in Table 3, and the 

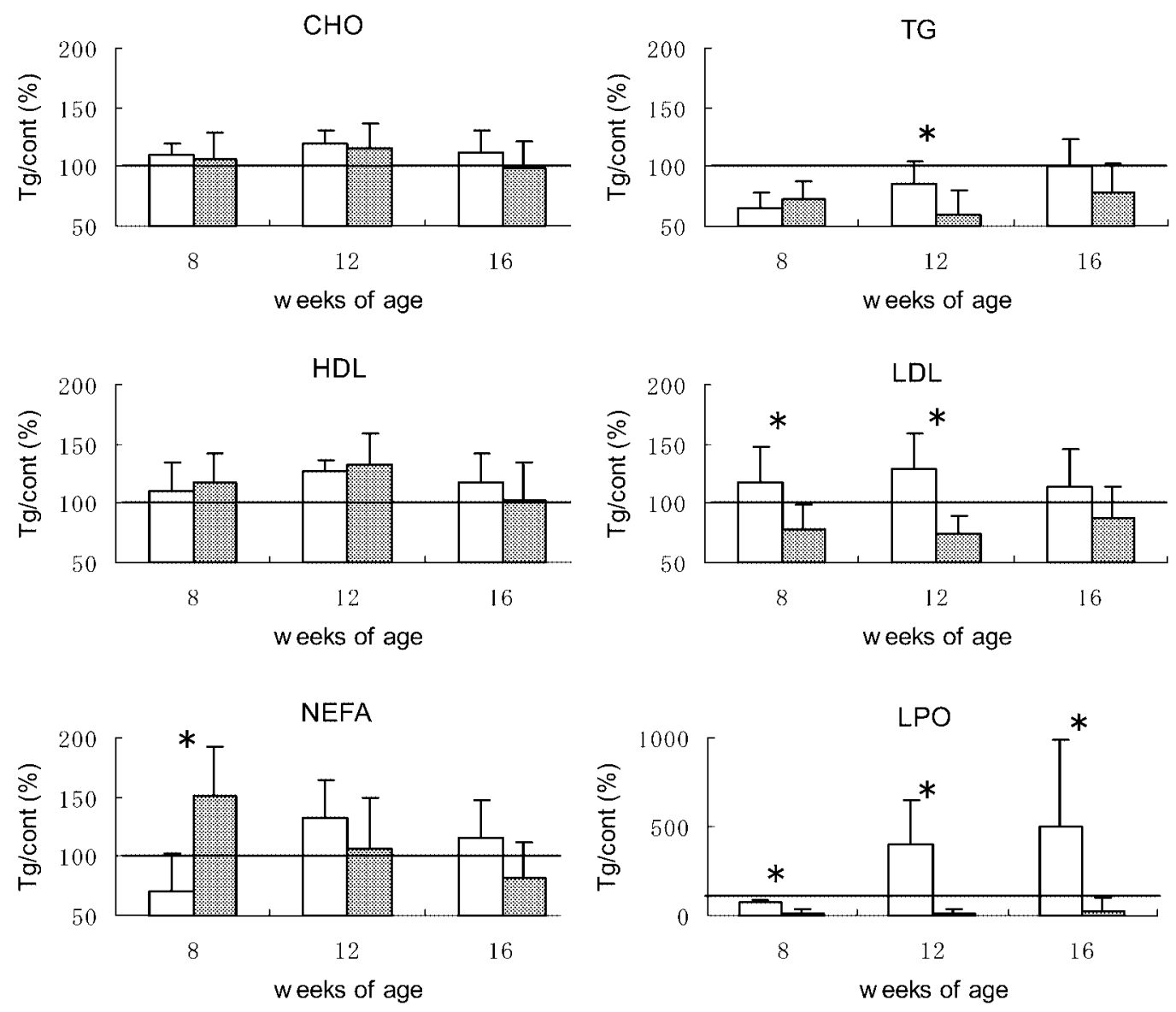

Fig. 2. Gender difference of cardiac risk biomarkers in transgenic mice. Each value for the transgenic females and males indicates the rate of increase or decrease (\%) relative to the value of the corresponding control mice (Tg/cont). The rates (\%) indicate the mean \pm S.D. CHO, total cholesterol; TG, triglyceride; HDL, HDL cholesterol; LDL, LDL cholesterol; NEFA, non esterified fatty acids; LPO, lipid peroxides. *: $P<0.05$ between transgenic females and transgenic males; filled column, females; open column, males.

corresponding micrographs are shown in Fig. 3.

In the livers of the 12-week-old transgenic males, hepatocytes with vacuolation indicative of lipid droplets were frequently observed in the centrilobular area as well as in the peri-lobular areas (Fig. 3-A), but this was not observed in the age-matched females (Fig. 3B). In the transgenic females, this abnormal feature was observed in only one animal at 16 weeks of age (Table 3).

In the hearts, there were no abnormalities in the control mice from 8 to 16 weeks of age (Fig. 3-C). In the transgenic mice, no histological abnormalities were observed until they reached 8 weeks of age. However, when they reached 12 weeks of age, a slight degeneration of the cardiomyocytes was observed in the hearts
Table 3. Histopathological data of the livers and hearts of transgenic mice

\begin{tabular}{|c|c|c|c|c|c|}
\hline \multirow{3}{*}{ Groups } & \multirow{3}{*}{$\begin{array}{c}\text { Weeks of } \\
\text { age }\end{array}$} & \multicolumn{2}{|c|}{ Liver } & \multicolumn{2}{|c|}{ Heart } \\
\hline & & \multicolumn{2}{|c|}{ Hepatic vacuolation } & \multicolumn{2}{|c|}{ Degeneration } \\
\hline & & Male & Female & Male & Female \\
\hline \multirow[t]{3}{*}{ Control } & 8 & 0 & 0 & 0 & 0 \\
\hline & 12 & 0 & 0 & 0 & 0 \\
\hline & 16 & 0 & 0 & 0 & 0 \\
\hline \multirow[t]{3}{*}{ Transgenic } & 8 & 0 & 0 & 0 & 0 \\
\hline & 12 & 2 & 0 & 3 & 2 \\
\hline & 16 & 3 & 1 & 3 & 3 \\
\hline
\end{tabular}

Three transgenic and three control mice were examined at each week of age. 

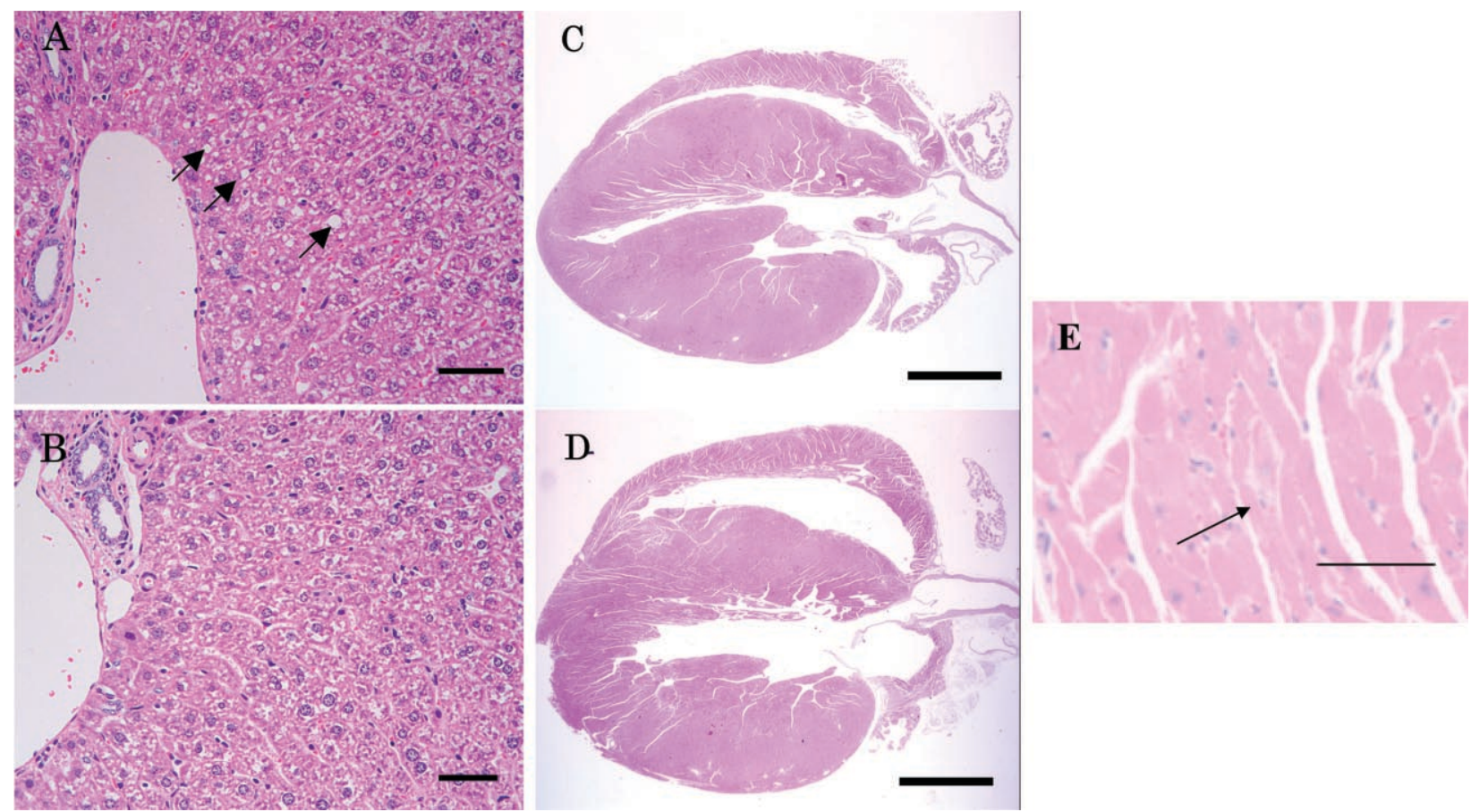

Fig. 3. Histopathology of the liver at 12 weeks of age and the heart at 16 weeks of age. A, liver of transgenic male; B, liver of transgenic female; C, heart of control male; D, heart of transgenic male; E, Magnification of D. The arrowheads in A denote vacuolation, indicative of lipid droplets. The arrow in $\mathrm{E}$ indicates regeneration in cardiomyocyte. The scale bars indicate $50 \mu \mathrm{m}$ in $\mathrm{A}, \mathrm{B}$, and $\mathrm{E}$, and $1 \mathrm{~mm}$ in $\mathrm{C}$ and $\mathrm{D}$.

of two transgenic females, and in those of all three transgenic males examined (Table 3-E). In addition, at 16 weeks of age, moderate cardiomegaly appeared in the hearts of the transgenic males (Table 1, and Fig. 3D). In both sexes at 16 weeks of age, however, the degree of degeneration was slight, as at 12 weeks of age.

\section{Discussion}

Transgenic laboratory animals with high levels of exogenous hGH have been widely used in the pathophysiological study of hormones. However, no studies of gender difference in the effects of the abnormal secretion of hGH on the cardiac function of transgenic animals have been carried out. The present transgenic mouse line expressing the CAG/EGFP-WAP/hGH cassette gene is a useful animal model, as it renders unnecessary routine DNA and RNA analyses of the transgene required for the maintenance of transgenic lines. This is possible because the transgenic offspring expressing the gene of interest (WAP/hGH) can be eas- ily identified by observation of body-surface fluorescence (due to the expression of EGFP), as shown in Fig. 1-B. In addition, it was reported that the transgenic rats and mice carrying the same $\mathrm{WAP} / \mathrm{hGH}$ transgene showed lower concentrations of endogenous GH owing to atrophy of GH secretion cells in the pituitary gland $[15,21,29]$, as compared to normal animals. Therefore, it is postulated that the transgenic mice in the present study also displayed a lower level of GH although we did not measure the concentration of mouse $\mathrm{GH}$.

In this study, we examined gender difference in biomarkers for cardiac risk caused by abnormal levels of hGH using a strain of transgenic mouse that exhibits the non-pulsatile secretion of plasma hGH. There was a significant increase in the mean body weight of the transgenic females, but not in that of transgenic males during the experiment, although the hGH levels of the transgenic females did not differ from those of the transgenic males. This gender difference was due to significantly increased tissue weights in the transgenic females. Gender difference was also observed in the 
blood levels of several biomarkers, indicating a risk of cardiac disorder. The most remarkable gender difference in the strain of transgenic mouse used in the present study was observed in the plasma LPO levels, and this difference was most notable in 8-week-old mice. LPO is formed as a product of oxidized unsaturated fatty acids and cholesterol, which are components of phospholipids and TG in the body.

It is well known that increases in plasma TG are due to lipid degradation caused by the anti-insulin effects of GH [10]. Therefore, it appears that the significant increase in LPO levels in the bloodstream of the transgenic males in this study was due to the abnormal secretion pattern of $\mathrm{hGH}$, thus indicating an additional effect of hGH on lipid metabolism and antioxidative capacity. Increases in LPO levels have been reported to impair the cell membranes of the vascular endothelium, resulting in the onset of arteriosclerosis [9, 12]. In addition, high levels of LPO in the blood have been observed in patients with myocardial infarction [23]. Based on these findings, as well as on observations that males commonly have higher levels of unsaturated fatty acids which serve as substrates for lipid oxidation than females [1], the abnormal secretion of hGH observed in the transgenic males in the present study may be, at least in part, responsible for the observed increase in LPO levels.

A higher incidence of lipid droplets in the liver was observed in the transgenic males at 16 weeks of age. All of the 16-week-old transgenic males exhibited vacuolation indicative of the accumulation of lipids in the liver, whereas only one transgenic female exhibited this abnormality. It is known that GH is secreted in a pulselike manner in mammals, and it is also known that there is a gender difference in the pattern of the secretion of GH [19], which in turn gives rise to metabolic differences between males and females. It has been reported that the pulsatile secretion pattern of endogenous GH was disrupted in transgenic rats expressing hGH under the control of the WAP promoter, which is the same promoter as that in the transgenic mice in the present study [14, 15]. In the livers of these rats, lipid accumulation and other metabolic abnormalities have been observed that lead to changes in microsomes CYP4A1 and CYP2E1, which are involved in lipid metabolism in the liver [30]. A high incidence of lipid droplets (vacuolation) in the liver of transgenic males may indicate the inadequate processing of excess fatty acids, which thereby accumulate in excess in the transgenic liver.

The transgenic males in the present study also exhibited higher levels of TG with increasing age, as compared to the pattern observed in the transgenic females. It has been suggested that hypertriglyceridemia in acromegaly is mediated, in part, by a decline in lipoprotein lipase and possibly by a decline in hepatic triglyceride lipase activity (HTGL) [20, 24]. The findings of these studies support our results showing elevated TG levels only in the transgenic males. The results obtained with the transgenic males in the present study may suggest that the lack of a male-specific hGH secretion pattern leads to decreases in HTGL activity, particularly among males.

Hauck et al. [11] reported that glutathione peroxidase (GPx) activity, catalase activity, and $\mathrm{Cu}-\mathrm{Zn}$ superoxide dismutase (SOD) activity in the liver, as well as GPx activity in the kidneys, were decreased in hGH-transgenic mice. In addition, GPx activity was reported to be higher in females than in males [13]. These findings suggest the possibility that the oxidation of lipids might have been accelerated by the abnormal secretion of hGH in our transgenic males. In addition, the result that a significant increase in LPO was observed only in the transgenic males may be explained by the antioxidative effects of estrogen. It has also been reported that arteriosclerosis is inhibited by the antioxidative effects of estrogen [32], and endothelial cell-protective action [6]. These reported findings may account for why no significant effects of abnormal hGH secretion on cardiac and liver function were observed in the transgenic females in the present study.

In the present study, a gender difference in LDL cholesterol levels was also observed in the transgenic mice. The rate of increase in LDL levels was higher in the transgenic males than in the transgenic females with advancing age. Elevated levels of LDL cholesterol have been observed in male patients with acromegaly [4]. In other GH-transgenic mice, LDL cholesterol was also increased due to a decrease in HTGL mRNA [28], which is known to be involved in LDL cholesterol uptake in the liver [8]. These findings suggest that elevated LDL cholesterol levels found in the transgenic males in the present study were due to decreases in HTGL activity. In addition, estrogen is known to in- 
duce the appearance of LDL receptors in the liver [27].

In this study, we did not histopathologically examine the blood vessels of the transgenic males. It has been reported that LOX-1 binds to oxidized LDL [31], and increases in oxidized LDL levels of expression are indicative of cardiovascular dysfunction [18]. We therefore analyzed LOX-1 mRNA expressions in the hearts of the transgenic mice, but no difference in their levels of expression was observed (data not shown). Therefore, we consider that there may have been no histopathological changes in the vascular system in the transgenic males in the present study. However, this study is the first to show the gender difference of cardiac risk biomarkers in $\mathrm{hGH}$ transgenic mice and this mice line will be useful model for studying gender difference caused by chronic-exposure of hGH. To gain a better understanding of the true relationship between excess hGH and cardiovascular function, further studies using older hGH-transgenic mice will be necessary.

In conclusion, we demonstrated in this study using hGH-transgenic mice that abnormal lipid metabolism and antioxidant ability were present, and in particular, the LPO levels were significantly increased in the transgenic males by the continuous secretion of $\mathrm{hGH}$, leading to a risk of cardiac dysfunction. The present results provide useful information regarding the use of $\mathrm{hGH}$ as a chronic medication in human males.

\section{Acknowledgments}

The authors would like to acknowledge Drs. Shinji Usuda, Toshiharu Sakai, Hideaki Okamiya and Hajime Tabata for their helpful support, as well as Mieko Saita and Nami Nagamatsu for their technical assistance and support. This work was supported in part by Grants-inAid for Scientific Research (Nos. 1436174 and 1465101) from the Ministry of Education, Culture, Sports, Science and Technology of Japan.

\section{References}

1. Aftergood, L. and Alfin-slater, R.B. 1965. Dietary and gonadal hormone effects on lipid metabolism in the rat. $J$. Lipid. Res. 58: 287-294.

2. Barnett-Connor, E. and Bush, T.L. 1991. Estrogen and coronary heart disease in women. JAMA 265: 1861-1867.

3. Bengtsson, B.A., Eden, S., Ernest, I., Oden, A., and Sjogren, B. 1988. Epidemiology and long-term survival in acromegaly. A study of 166 cases diagnosed between 1955 and 1984. Acta. Med. Scand. 223: 327-335.

4. Colao, A., Ferone, D., Marzullo, P., Cappabianca, P., Cirillo, S., Boerlin, V., Lancranjan, I., and Lombardi, G. 2001. Long-term effects of depot long-acting samatostatin analog octreotide on hormone levels and tumor mass in acromegaly. J. Clin. Endocrinol. Metab. 86: 2779-2786.

5. Eaker, E.D., Packard, B., and Thom, T.J. 1989. Epidemiology and risk factors for coronary heart disease in women. Cardiovasc. Clin. 19: 129-145.

6. Farhat, M.Y., Lavigne, M.C., and Ramwell, P.W. 1996. The vascular protective effects of estrogen. FASEB. J. 10: 615-624.

7. Fazio, S., Sabatini, D., Capaldo, B., Vigorito, C., Giordano, A., Guida, R., Pardo, F., Biondi, B., and Sacca, L. 1996. A preliminary study of growth hormone in the treatment of dilated cardiomyopathy. N. Engl. J. Med. 334: 809-814.

8. Frick, F., Bohlooly-Y, M., Linden, D., Olsson, B., Tornell, J., Eden, S., and Oscarsson, J. 2001. Long-term growth hormone excess induces marked alterations in lipoprotein metabolism in mice. Am. J. Physiol. Endocrinol. Metab. 281: E1230-1239.

9. Fukuzumi, K. 1986. Relationship between lipoperoxides and diseases. J. Environ. Pathol. Toxicol. Oncol. 6: 25-56.

10. Harant, I., Beauville, M., Crampes, F., Riviere, D., Tauber, M.T., Tauber, J.P., and Garrigues, M. 1994. Response of fat cells to growth hormone $(\mathrm{GH})$ : effect of long term treatment with recombinant human GH in GH-deficient adults. 1994. J. Clin. Endocrinol. Metab. 78: 1392-1395.

11. Hauck, S.J. and Bartke, A. 2001. Free radical defenses in the liver and kidney of human growth hormone transgenic mice: possible mechanisms of early mortality. J. Gerontol. A. Biol. Sci. Med. Sci. 56: B153-B162.

12. Henning, B. and Chow, C.K. 1988. Lipid peroxidation and endothelial cell injury: implications in atherosclerosis. Free. Radic. Biol. Med. 4: 99-106.

13. Igarashi, T., Satoh, T., Ueno, K., and Kitagawa, H. 1983. Sex-related difference in the hepatic glutathione level and related enzyme activities in rat. J. Biochem. 93: 33-36.

14. Ikeda, A., Chang, K.T., Matsumoto, Y., Furuhata, Y., Nishihara, M., and Takahashi, M. 1998. Obesity and insulin resistance in human growth hormone transgenic rats. Endocrinology. 139: 3057-3063.

15. Ikeda, A., Matsumoto, Y., Chang, K.T., Nakano, T., Matsuyama, S., Yamanouchi, K., Ohta, A., Nishihara, M., Tojo, H., Sasaki, F., and Takahashi, M. 1997. Different female reproductive phenotypes determined by human growth hormone (hGH) levels in hGH-transgenic rats. Biol. Reprod. 56: 847-851.

16. Kornstein, S.G. and McEnany, G. 2000. Enhancing pharmacologic effects in the treatment of depression in women. J. Clin. Psychiatry. 61: 18-27.

17. Kubo, J., Yamanouchi, K., Naito, K., and Tojo, H. 2002. Expression of the gene of interest fused to the EGFPexpressing gene in transgenic mice derived from selected transgenic embryos. J. Exp. Zool. 293: 712-718.

18. Kume, N. and Kita, T. 2001. Lectin-like oxidized lowdensity lipoprotein receptor-1 (LOX-1) in atherogenesis. Trends. Cardiovasc. Med. 11: 22-25. 
19. Macleod, J.N., Pampori, N.A., and Shapiro, B.H. 1991. Sex differences in the ultradian pattern of plasma growth hormone concentrations in mice. J. Endocrinol. 131: 395399.

20. Murase, T., Yamada, N., Ohsawa, N., Kosaka, K., Morita, S., and Yoshida, S. 1980. Decline of postheparin plasma lipoprotein lipase in acromegalic patients. Metabolism. 29: 666-672.

21. Nagasawa, H., Hasegawa, M., Yamamoto, K., Sakamoto, S., Mori, T., and Tojo, H. 1993. Normal and neoplastic growth of mammary glands and circulation levels of prolactin and growth hormone in mouse whey acidic protein promoter/human growth hormone (mWAP/hGH) transgenic mice. Zool. Sci. 10: 963-970.

22. Niwa, H., Yamamura, K., and Miyazaki, J. 1991. Efficient selection for high-expression transfectants with a novel eukaryotic vector. Gene 108: 193-209.

23. Oen, L.H., Utomo, H., Suyatna, F., Hanafiah, A., and Asikin, N. 1992. Plasma lipid peroxides in coronary heart disease. Int. J. Clin. Pharmacol. Ther. Toxicol. 30: 77-80.

24. Olsson, B., Bohlooly-Y, M., Brusehed, O., Isaksson, O.G., Ahren, B., Olofsson, S.O., Oscarsson, J., and Tornell, J. 2003. Bovine growth hormone-transgenic mice have major alteration in hapatic expression of metabolic genes. Am. J. Physiol. Endocrinol. Metab. 285: E504-E511.

25. Rademaker, M. 2001. Do women have more adverse drug reactions? Am. J. Clin. Dermatol. 2: 349-351.

26. Rosen, T. and Bengtsson, B.A. 1990. Premature mortality due to cardiovascular disease in hypopituitarism. Lancet.
336: 285-288.

27. Sacks, F.M. and Walsh, B.W. 1990. The effects of reproductive hormones on serum lipoproteins: unresolved issues in biology and clinical practice. Ann. N. Y. Acad. Sci. 592: 272-285.

28. Santamarina-Fojo, S., Haudenschild, C., and Amar, M. 1998. The role of hepatic lipase in lipoprotein metabolism and atherosclerosis. Curr. Opin. Lipidol. 9: 211-219.

29. Sasaki, F., Tojo, H., Iwama, Y., Miki, N., Meada, K., Ono, M., Kiso, Y., Okada, T., Matsumoto, Y., and Tachi, C. 1997. Growth hormone releasing hormone (GHRH) - GHsomatic growth and luteinizing hormone (LH) RH-ovarian axes in adult female transgenic mice expressing human GH gene. J. Neuroendocri. 9: 615-626.

30. Takahashi, J., Furuhata, Y., Ikeda, A., Takahashi, M., Iwata, H., Kazusaka, A., and Fujita, A. 1999. Characterization of hepatic cytochrome $\mathrm{P} 450$ isozyme composition in the transgenic rat expressing low level human growth hormone. Xenobiotica. 29: 1203-1212.

31. Toshima, S., Hasegawa, A., Kurabayashi, M., Itabe, H., Takano, T., Sugano, J., Shimamura, K., Kimura, J., Michishita, I., Suzuki, T., and Nagai, R. 2000. Circulation oxidized low density lipoprotein levels. A biochemical risk marker for coronary heart disease. Arterioscler. Thromb. Vasc. Biol. 20: 2243-2247.

32. Wolinsky, H. 1972. Effects of estrogen and progesterone treatment on the response of the aorta of male rats to hypertension. Circ. Res. 30: 341-349. 